\title{
Trichobezoar presenting as an epigastric tumour
}

\author{
Deepthi Samarage $^{1}$, Malik Samarasinghe ${ }^{2}$, S I Samarasekera ${ }^{3}$ \\ Sri Lanka Journal of Child Health, 2004; 34: 60-1
}

(Key words: Trichobezoar, epigastric tumour)

\section{Introduction}

Bezoar is an accumulation of exogenous matter in stomach or intestine ${ }^{1}$. Trichobezoar literally means a 'hairball', a collection of the patient's own hair entwined with undigested food ${ }^{1,2}$. Trichotelemania means pulling at one's own hair and trichophagia means ingestion of hair. They are closely related and can lead to trichobezoar ${ }^{2}$.

\section{Case report}

A previously healthy $81 / 2$ year old girl from Mount Lavinia was admitted to a paediatric unit, Colombo South Teaching Hospital, in August 2004 with a history of recurrent abdominal pain and vomiting of two months duration. The pain was of moderate severity, aching in nature and mainly epigastric. It was associated with mild abdominal distension, usually after meals, resolving with vomiting. Her bowel habits and appetite were normal and there was no recent weight loss. She did not give any history of pica. There were no significant illnesses in the past. Birth and developmental histories were normal. She is a year 4 student with an average school performance. There was no family history of psychiatric disorders

Examination revealed a well looking child with a height of $115.5 \mathrm{~cm}$ (2nd centile) and a weight of 18.2 $\mathrm{kg}$ (2nd-9th centile). She was not pale or icteric but was irritable and had a poor attention span. Hair was scanty, but no bald patches were noted. Abdomen was soft and non tender. There was a firm, ill defined mass, $5 \mathrm{~cm}$ by $10 \mathrm{~cm}$, in the epigastric region which was attached to deep structures. Liver and spleen were not palpable and no free fluid was detected in the abdomen. She was otherwise clinically normal.

${ }^{1}$ Consultant Paediatrician, Colombo South Teaching Hospital, ${ }^{2}$ Consultant Paediatric Surgeon, Lady Ridgeway Hospital, ${ }^{3}$ Registrar in Paediatrics, Colombo South Teaching Hospital.

(Received on 6 December 2004)
An ultrasound scan (USS) of the abdomen showed an ill defined solid mass in the epigastrium, consistent with stomach, not attached to surrounding structures. No enlarged paravertebral lymph nodes were seen and the liver and spleen were normal. There was no free fluid in the abdomen.

She was transferred to a paediatric surgical unit at the Lady Ridgeway Hospital where a CT scan of the abdomen was done which revealed a well defined, solid mass, $5 \mathrm{~cm} \mathrm{x} 10 \mathrm{~cm}$, in the stomach. Margins were clearly demarcated from the walls of stomach. Appearance was suggestive of a trichobezoar (Figure 1).

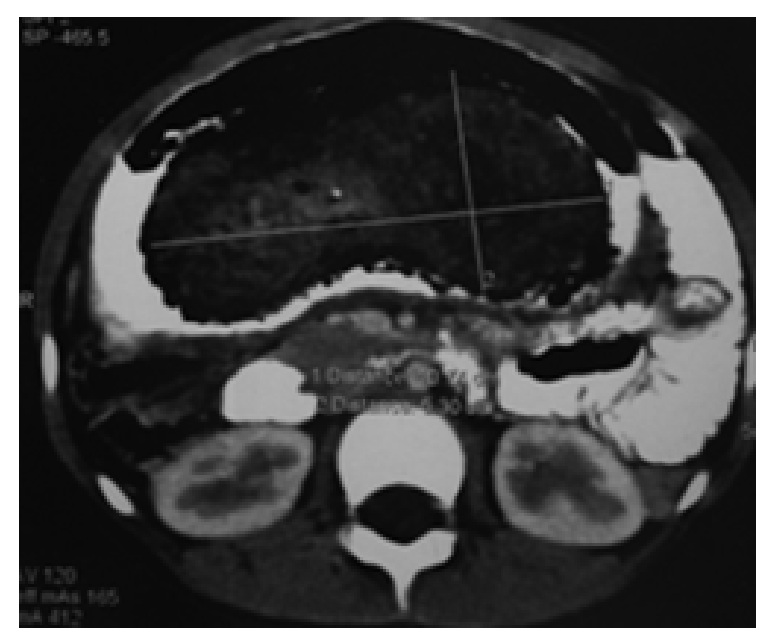

Figure $1 C T$ scan of abdomen showing mass in stomach

She was subjected to an upper GI endoscopy and a 'hairball' was visualised in the stomach. There were no associated gastric erosions or ulcers. Endoscopic removal of the 'hairball' was unsuccessful because it was huge. She subsequently underwent a laparotomy and the hairball was removed (Figure 2). She had an uneventful postoperative recovery. Subsequent psychiatric examination revealed a mild personality disorder for which she is been followed up. 


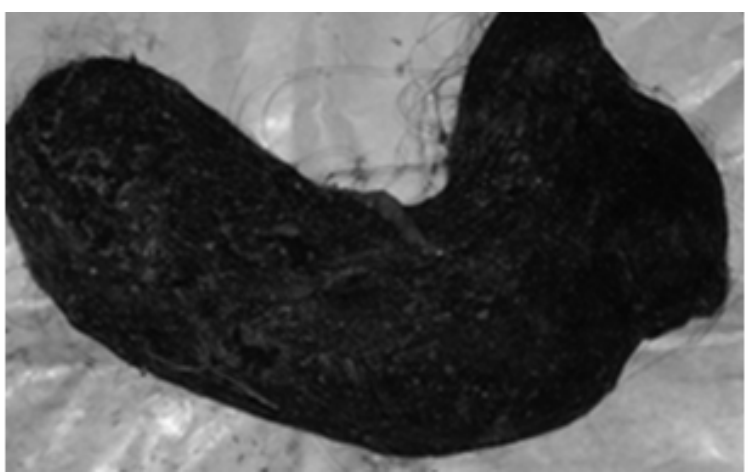

Figure 2 showing the removed 'hairball'

\section{Discussion}

Trichobezoar (hair ball) is a rare condition which occurs mostly in females with personality or neurological disorders with a peak incidence in the 2 nd decade of life ${ }^{1,5}$. Trichobezoars can become large and may form a cast in the stomach ${ }^{1,2}$ sometimes extending the tail into the proximal part of the duodenum (Rapunzel syndrome) ${ }^{2,6}$.

Presenting symptoms are related to gastric outlet obstruction or partial intestinal obstruction including intermittent vomiting, anorexia and weight loss ${ }^{1,2}$. Patient may complain of abdominal pain, distension, haematemesis and halitosis ${ }^{1}$. Examination findings are patchy baldness and firm left hypochondrial mass ${ }^{1,2}$. Associated chronic gastritis can cause iron deficiency anaemia, hypoproteinaemia, and steatorrohea $^{1,4}$.

Plain x-ray of abdomen, USS of abdomen and barium examination are supportive investigations ${ }^{1,3,4}$. Upper gastrointestinal endoscopy is the definitive diagnostic test and it is therapeutic as well in disrupting and removing the bezoar ${ }^{1,3}$. If endoscopic removal fails, surgical intervention may become necessary to relieve obstruction and necrosis ${ }^{1,2}$. A multidisciplinary approach should be adopted to prevent recurrence ${ }^{2}$. In a patient with learning difficulties and gastrointestinal symptoms a low threshold for upper gastrointestinal endoscpy should be maintained ${ }^{2}$.

\section{References}

1. Robert W. Foreign bodies and bezoars. In: Behrmann R E, Kliegman R M, Arvin A W, editors, Nelson Textbook of Paediatrics. London: W B Saunders, 2000; 1144.

2. Ramadan N, Pandya N A, Bhaduri B. A Rapunzel with a differnce. Archives of Diseases in childhood 2003; 88 (3): 264.

3. Russell, Williams, Bulstrode: Bailey and Love's Short Practice of Surgery: 23rd Edition. London: Chapman \& Hall 2000.

4. Byrne W J: Bezoars. Gastrointestinal Endoscopic North American Clinic Notes 1994; 4:99.

5. Christensos G A, Mackenzie T B, Michell J E, et al. Characteristics of 60 adult chronic hair pullers. AMJ in Psychiatry 1991; 148:365-70.

6. Singla S Z, Rattan K N, Kaushi K N, et al. Rapunzel - a case report. AMJ in Gastroenterol 1999; 94: 1970-1. 
